\title{
Determination of an Effective Detector Position for Pulsed-Neutron-Source Alpha Measurement by Time-Dependent Monte Carlo Neutron Transport Simulations
}

\author{
Sang Hoon Jang $(\mathbb{D}$ and Hyung Jin Shim $\mathbb{D}$ \\ Seoul National University, 1 Gwanak-ro, Gwanak-gu, Seoul 08826, Republic of Korea \\ Correspondence should be addressed to Hyung Jin Shim; shimhj@snu.ac.kr
}

Received 31 January 2018; Accepted 15 March 2018; Published 2 May 2018

Academic Editor: Eugenijus Ušpuras

Copyright (C) 2018 Sang Hoon Jang and Hyung Jin Shim. This is an open access article distributed under the Creative Commons Attribution License, which permits unrestricted use, distribution, and reproduction in any medium, provided the original work is properly cited.

\begin{abstract}
A simple method using the time-dependent Monte Carlo (TDMC) neutron transport calculation is presented to determine an effective detector position for the prompt neutron decay constant $(\alpha)$ measurement through the pulsed-neutron-source (PNS) experiment. In the proposed method, the optimum detector position is searched by comparing amplitudes of detector signals at different positions when their $\alpha$ estimates by the slope fitting are converged. The developed method is applied to the $\mathrm{Pb}$ - $\mathrm{Bi}$ zoned ADS experimental benchmark at Kyoto University Critical Assembly. The $\alpha$ convergence time estimated by the TDMC PNS simulation agrees well with the experimental results. The $\alpha$ convergence time map and the corresponding signal amplitude map predicted by the developed method show that polyethylene moderator regions adjacent to fuel region are better positions than other candidates for the PNS $\alpha$ measurement.
\end{abstract}

\section{Introduction}

Since the early 1990s, accelerator-driven subcritical systems (ADS) for transmutation of radioactive wastes and energy production have been proposed and designed throughout the world with their advantages of high flexibility of fuel compositions and the enhanced safety concept [1-3]. The neutronic characteristics of the subcritical reactor have been extensively studied theoretically $[4,5]$ and experimentally [68]. The prompt neutron decay constant (hereafter referred to as $\alpha$ ) of a subcritical system is a fundamental kinetics parameter which represents its asymptotic behavior ignoring the delayed neutron effect. Moreover $\alpha$ can be directly measured $[9,10]$ by injecting a short burst of neutrons in the system, called the pulsed-neutron-source (PNS) experiment. Since Simmons and King [9] applied an exponential regression to neutron detector signals from the PNS experiment, this $\alpha$ measurement method has been popularly employed because it can provide $\alpha$ results independent of the positioning and energy characteristics of the detector and neutron source
$[9,11,12]$ by reducing higher-mode contaminations on the exponential fitting $[13,14]$.

In practice, however, the PNS $\alpha$ measurement may yield considerably different results at different detector positions and neutron sources, as reported in the experimental benchmarks on an ADS at Kyoto University Critical Assembly (KUCA) [15, 16]. This measurement dependency on the detector position and the neutron source can be attributed mostly to the signal contamination $[11,16]$ by the highermode components of the prompt neutron flux, which is caused by taking detector signals before the higher-mode components fully decay out. It is difficult, however, to obtain confident detector signals after the prompt neutron flux converges to the fundamental mode in a deep subcritical system where the prompt neutron flux decreases rapidly. Therefore, it is necessary to determine effective detector positions where the prompt neutron flux converges fast with larger signal strength than other candidate positions.

The objective of this paper is to devise a simple but practical way to determine an optimum detector position 
for the $\alpha$ measurement through the PNS experiment using the time-dependent Monte Carlo (TDMC) neutron transport analyses [17-19]. In the TDMC calculations, the combing algorithm $[17,20]$ is applied to maintain the time-bin-wise neutron population because an exponential decrease of the neutron population in an analog TDMC calculation of a subcritical system causes large statistical uncertainties. In the proposed method, the optimum detector position is searched by comparing the strength of detector signal at each spatial position when the $\alpha$ estimate at the position is converged. The position-dependent $\alpha$ convergence is diagnosed by a slope fitting to the detector signals obtained from the TDMC calculations. The proposed methods are implemented in a Seoul National University continuous-energy Monte Carlo (MC) code, McCARD [21], and applied to the Pb-Bi-zoned ADS experimental benchmark at KUCA [22].

\section{Determination of an Optimum Detector Position through the TDMC Analysis}

2.1. TDMC PNS Simulation. The population of prompt neutrons induced from a fast neutron burst in a subcritical system decreases exponentially. Thus a special population control technique is necessary for an efficient TDMC calculation. Here we adopt an analog MC simulation of the branching process in which extra neutrons from fission are sampled and tracked accompanied with the combing technique [17]. In the TDMC simulations with the combing technique, the time domain is split into time bins and each neutron is simulated time-bin-by-time-bin with updating its time variable whenever its track is sampled by [19]

$$
t_{k}^{i, j}=t_{k-1}^{i, j}+\frac{l_{k}^{i, j}}{\sqrt{2 E_{k}^{i, j} / m_{n}}},
$$

where $t_{k^{\prime}}^{i, j}\left(k^{\prime}=k\right.$ or $\left.k-1\right), l_{k}^{i, j}$, and $E_{k}^{i, j}$ are the time after the $k^{\prime}$ th flight, the length, and the neutron energy of the $k$ th track of history $j$ at time bin $i . m_{n}$ is the neutron mass. If the sampled time is greater than the upper time bound of the $i$ th time bin, that is, $t_{k}^{i, j}>T_{i+1}$, then the track length of and time after the last flight $K$ of history $j$, denoted by $l_{K}^{i, j}$ and $t_{K}^{i, j}$, respectively, become

$$
\begin{aligned}
& l_{K}^{i, j}=\left(T_{i+1}-t_{K-1}^{i, j}\right) \cdot \sqrt{\frac{2 E_{K}^{i, j}}{m_{n}}}, \\
& t_{K}^{i, j}=T_{i+1},
\end{aligned}
$$

where $E_{K}^{i, j}$ means the neutron energy of the last flight $K$ of history $j$ at time bin $i$. After the $i$ th time-bin TDMC simulations for all histories, the number of neutrons for the next time-bin simulations is increased to be the user-inputted number of histories by splitting according to the number of surviving neutrons at $T_{i+1}$ with conserving the total weight.

2.2. $\alpha$ Estimation by the Slope Fitting. The time-dependent detector signals from prompt neutrons can be represented by $\mathrm{MC}$ responses of the reaction rate in the detector volume $V_{D}$ at $\mathbf{r}$ during time interval $\left(t_{i^{\prime}}-\Delta t / 2, t_{i^{\prime}}+\Delta t / 2\right), R_{D}\left(\mathbf{r}, t_{i^{\prime}}\right)$, defined as

$$
\begin{aligned}
& R_{D}\left(\mathbf{r}, t_{i^{\prime}}\right) \\
& \quad=\sum_{m} \sum_{r} \int_{V_{D}} \int_{E} \int_{t_{i^{\prime}}-\Delta t / 2}^{t_{i^{\prime}}+\Delta t / 2} \sum_{r}^{m}(\mathbf{r}, E) \phi_{p}(\mathbf{r}, E, t) d \mathbf{r} d E d t,
\end{aligned}
$$

where $i^{\prime}, m$, and $r$ are the time-step, isotope, and reaction type index. $\phi_{p}$ denotes the prompt neutron flux.

Then $\alpha$ corresponding to the detector position $\mathbf{r}$ can be estimated by an exponential fitting to the TDMC results of $R_{D}\left(\mathbf{r}, t_{i^{\prime}}\right)$ as [13]

$$
R_{D}(\mathbf{r}, t)=C_{1} \cdot \exp \left[-\alpha_{\mathrm{est}}\left(\mathbf{r} \mid t_{s}\right) \cdot\left(t-t_{s}\right)\right]+C_{2},
$$

where $C_{1}$ and $C_{2}$ are fitting constants and $t$ and $t_{s}$ are the time after the neutron burst and the beginning time of the fitting interval, respectively. $\alpha_{\text {est }}\left(\mathbf{r} \mid t_{s}\right)$ indicates an estimate of $\alpha$ from a neutron detector located at $\mathbf{r}$ using $t_{s}$. In this study, $\alpha_{\text {est }}\left(\mathbf{r} \mid t_{s}\right)$ are calculated with increasing $t_{s}$ from $0.0 \mathrm{~ms}$ to $3.9 \mathrm{~ms}$ by $0.1 \mathrm{~ms}$ and setting the fitting interval to $1.0 \mathrm{~ms}$.

An onset time of the convergence of $\alpha_{\text {est }}(\mathbf{r}), t_{0}(\mathbf{r})$ is determined when the relative error of a mean value of $\alpha_{\text {est }}(\mathbf{r})$ $t_{s}$ ) comparing to its reference, denoted by $\alpha_{\text {ref }}$, becomes less than a prescribed value $\varepsilon$ as

$$
\begin{aligned}
t_{0}(\mathbf{r}) & =\min \left\{t_{s} ;\left|\frac{\overline{\alpha_{\text {est }}}\left(\mathbf{r} \mid t_{s}\right)-\alpha_{\text {ref }}}{\alpha_{\text {ref }}}\right|<\varepsilon\right\} ; \\
\overline{\alpha_{\text {est }}}\left(\mathbf{r} \mid t_{s}\right) & =\frac{1}{N} \sum_{n=1}^{N} \alpha_{\text {est }, n}\left(\mathbf{r} \mid t_{s}\right),
\end{aligned}
$$

where $N$ is the number of replicas with different random number sequences. $\alpha_{\text {est } n}\left(\mathbf{r} \mid t_{s}\right)$ is an $\alpha$ estimate of the $n$th replica calculation. $\varepsilon$ of 0.05 is used for this convergence diagnosis.

Here $\alpha_{\text {ref }}$ is calculated by the MC $\alpha$-iteration method [23] which is developed to solve the $\alpha$-mode eigenvalue equation expressed as

$$
\begin{aligned}
& S_{t}=\alpha \mathbf{R} S_{t} ; \\
& \mathbf{R S}_{t} \equiv \frac{1}{v(E) \Sigma_{t}(\mathbf{r}, E)} \\
& \quad \cdot \sum_{k=0}^{\infty} \int d \mathbf{r}^{\prime} \int d E_{0} \int d \mathbf{\Omega}_{0} K_{p, k}\left(\mathbf{r}^{\prime}, E_{0}, \boldsymbol{\Omega}_{0} \longrightarrow \mathbf{r}, E, \mathbf{\Omega}\right) \\
& \quad \times \int d \mathbf{r}_{0} T\left(\mathbf{r}_{0} \longrightarrow \mathbf{r}^{\prime} \mid E_{0}, \boldsymbol{\Omega}_{0}\right) S_{t}\left(\mathbf{r}_{0}, E_{0}, \boldsymbol{\Omega}_{0}\right), \\
& K_{p, k}\left(\mathbf{r}^{\prime}, E_{0}, \mathbf{\Omega}_{0} \longrightarrow \mathbf{r}, E, \boldsymbol{\Omega}\right)=\int d \mathbf{r}_{1} \int d E_{1} \int d \mathbf{\Omega}_{1} \\
& \quad \ldots \int d \mathbf{r}_{k-1} \int d E_{k-1} \int d \mathbf{\Omega}_{k-1} \times K_{p}\left(\mathbf{r}_{k-1}, E_{k-1}, \mathbf{\Omega}_{k-1}\right. \\
& \quad \longrightarrow \mathbf{r}, E, \boldsymbol{\Omega}) \cdots K_{p}\left(\mathbf{r}^{\prime}, E_{0}, \mathbf{\Omega}_{0} \longrightarrow \mathbf{r}_{1}, E_{1}, \mathbf{\Omega}_{1}\right),
\end{aligned}
$$




$$
\begin{aligned}
& K_{p}\left(\mathbf{r}^{\prime}, E^{\prime}, \mathbf{\Omega}^{\prime} \longrightarrow \mathbf{r}, E, \boldsymbol{\Omega}\right)=T\left(\mathbf{r}^{\prime} \longrightarrow \mathbf{r} \mid E, \boldsymbol{\Omega}\right) \\
& \cdot C_{p}\left(E^{\prime}, \mathbf{\Omega}^{\prime} \longrightarrow E, \boldsymbol{\Omega} \mid \mathbf{r}^{\prime}\right), \\
& C_{p}\left(E^{\prime}, \mathbf{\Omega}^{\prime} \longrightarrow E, \boldsymbol{\Omega} \mid \mathbf{r}^{\prime}\right) \\
& \quad=\sum_{r \neq \text { fis. }} \frac{\nu_{r} \Sigma_{r}\left(\mathbf{r}^{\prime}, E^{\prime}\right)}{\Sigma_{t}\left(\mathbf{r}^{\prime}, E^{\prime}\right)} f_{r}\left(E^{\prime}, \mathbf{\Omega}^{\prime} \longrightarrow E, \boldsymbol{\Omega}\right) \\
& \quad+\frac{\nu_{p} \Sigma_{f}\left(\mathbf{r}^{\prime}, E^{\prime}\right)}{\sum_{t}\left(\mathbf{r}^{\prime}, E^{\prime}\right)} \cdot \frac{\chi_{p}(E)}{4 \pi}, \\
& T\left(\mathbf{r}^{\prime} \longrightarrow \mathbf{r}^{\prime} E, \boldsymbol{\Omega}\right)=\frac{\Sigma_{t}(\mathbf{r}, E)}{\left|\mathbf{r}-\mathbf{r}^{\prime}\right|^{2}} \\
& \left.\quad \cdot \exp -\int_{0}^{\left|\mathbf{r}-\mathbf{r}^{\prime}\right|} \Sigma_{t}\left(\mathbf{r}-s \frac{\mathbf{r}-\mathbf{r}^{\prime}}{\left|\mathbf{r}-\mathbf{r}^{\prime}\right|}, E\right) d s\right] \delta(\boldsymbol{\Omega} \\
& \left.\quad \cdot \frac{\mathbf{r}-\mathbf{r}^{\prime}}{\left|\mathbf{r}-\mathbf{r}^{\prime}\right|}-1\right), \\
& S_{t} \equiv \frac{\alpha}{v(E)} \phi_{p}(\mathbf{r}, E, \boldsymbol{\Omega}),
\end{aligned}
$$

where the subscript $p$ indicates prompt neutron. $S_{t}$ is named the time source [23]. $v(E)$ is a neutron speed corresponding to its energy E. $v_{r}$ and $v_{p}$ denote the average numbers of neutrons emitted from reaction type $r$ and prompt fission neutrons, respectively. $f_{r}\left(E^{\prime}, \boldsymbol{\Omega}^{\prime} \rightarrow \mathrm{E}, \boldsymbol{\Omega}\right) d E d \boldsymbol{\Omega}$ is the probability that a collision of type $r$ by a neutron of direction $\boldsymbol{\Omega}^{\prime}$ and energy $E^{\prime}$ will produce a neutron in direction interval $d \boldsymbol{\Omega}$ about $\boldsymbol{\Omega}$ with energy in $d E$ about $E$. Other notations follow convention. By directly applying the power iteration method [24] for (8), it is demonstrated [23] to stably estimate $\alpha$ even for a deep subcritical system.

2.3. Determination of an Optimum Detector Position. The amplitude of neutron signals used for the exponential regression when $\alpha_{\text {est }}(\mathbf{r})$ is converged can be defined as

$$
\begin{aligned}
& \overline{R_{D}}(\mathbf{r}) \\
& =\sum_{m} \sum_{r} \int_{V_{D}} \int_{E} \int_{t_{0}(\mathbf{r})}^{t_{0}(\mathbf{r})+\Delta T} \sum_{r}^{m}(\mathbf{r}, E) \phi_{p}(\mathbf{r}, E, t) d \mathbf{r} d E d t,
\end{aligned}
$$

where $\Delta T$ denotes the fitting time interval.

Then the optimum detector position for the PNS $\alpha$ measurement can be determined as a position $\mathbf{r}$ where $\overline{R_{D}}(\mathbf{r})$ becomes maximized because the statistical uncertainty of the detector signals during $\left[t_{0}(\mathbf{r}), t_{0}(\mathbf{r})+\Delta T\right]$ is assumed to be inversely proportional to the signal amplitude at the position by following the Poisson distribution.

\section{Application Results}

3.1. Pb-Bi-Zoned Experimental Benchmark. The developed method to determine the optimum detector position for the PNS $\alpha$ measurement is applied for the $\mathrm{Pb}$-Bi-zoned ADS experimental benchmark at KUCA [22]. The benchmark

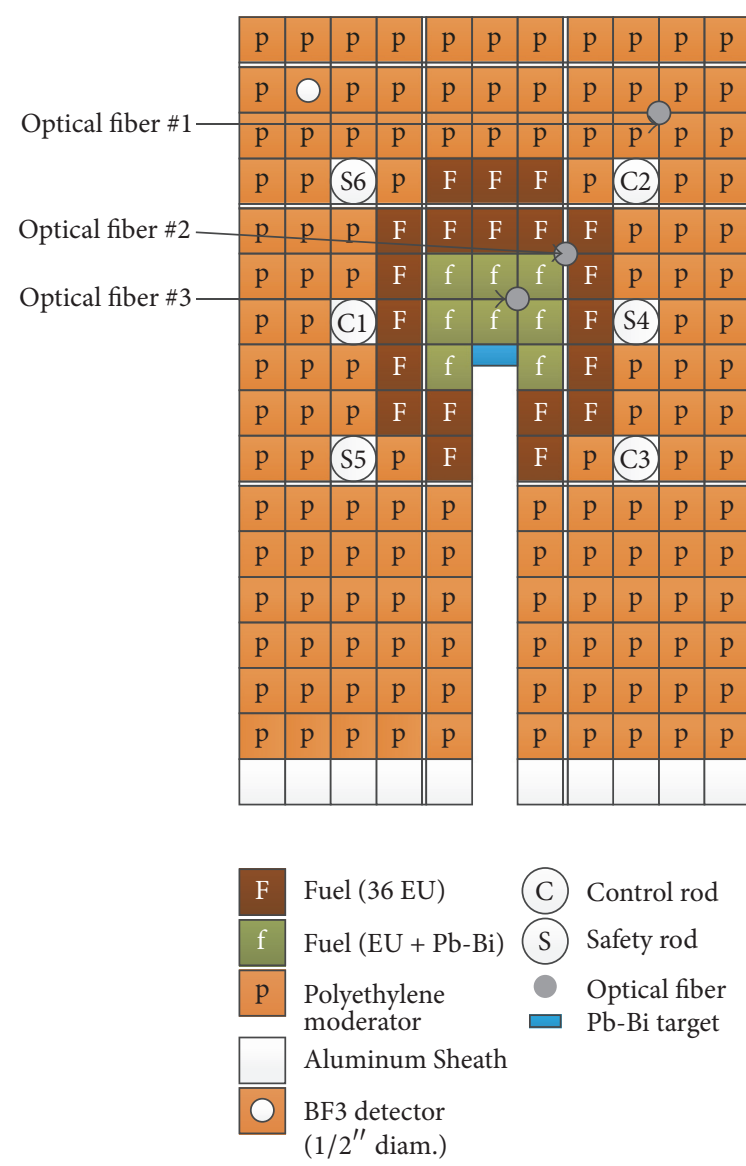

Figure 1: Core configuration of the PNS experiment (Case 6).

provides 6 different subcritical cores comprised of $\mathrm{Pb}-\mathrm{Bi}$ loaded enriched uranium fuel and polyethylene moderator and reflector. The spallation neutron source is generated in the center of the core by injecting $100 \mathrm{MeV}$ protons to the $\mathrm{Pb}$ Bi target. The PNS $\alpha$ measurement is conducted with three optical fiber detectors in different positions. Case 6 among the six cores is chosen for an application of the developed method and its core configuration is shown in Figure 1.

3.1.1. Spallation Source Treatment. The spallation neutron source information is obtained from MCNPX2.6.0 [25] proton source simulations. The spallation neutron spectra from the $\mathrm{Pb}-\mathrm{Bi}$ target are tallied with respect to angle between the outgoing direction of neutrons and proton beam. The angle bin is equally divided by 15 degrees. The MCNPX calculation is done with 10,000,000 histories and la150h proton library provided. The neutron spectra and relative angular flux distribution are given in Figure 2. One can see that the neutron spectra tend to be more hardened as its direction is more forwarded and the overall neutron yield is biased to the forward direction. The direction and energy of the spallation neutrons are inputted in the form of histograms and uniformly sampled in each bin at the beginning of the McCARD TDMC simulations. 


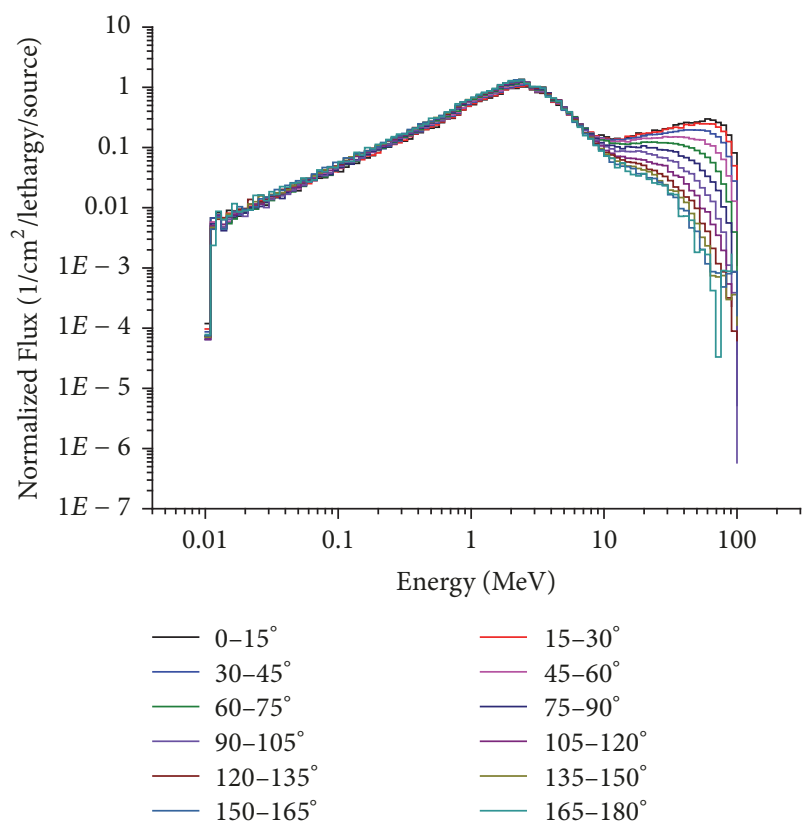

(a)

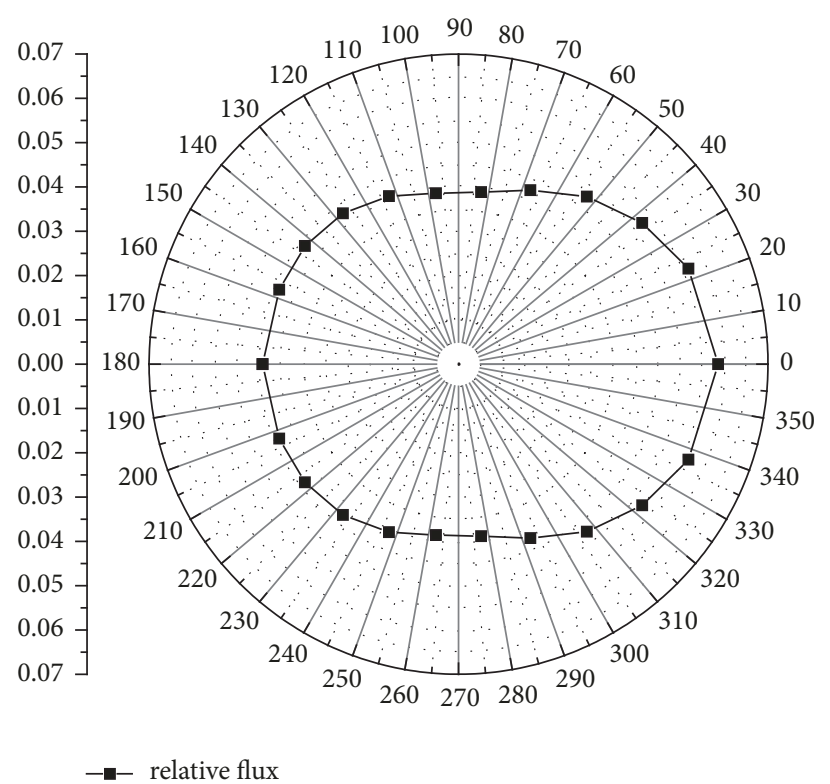

(b)

FIgURE 2: Spallation neutron spectra (a) and relative angular flux distribution (b).

3.1.2. Detector Modeling. The neutron detector used in the experiment is a small-sized optical fiber detector [26] with $1 \mathrm{~mm}$ diameter which makes it available to be inserted into gaps between assemblies. The detector consists of a mixture of ${ }^{6} \mathrm{LiF}$ neutron converter and $\mathrm{ZnS}$ scintillator of which signals are induced by charged particles emitted from $(n, \alpha)$ and $(n, p)$ reactions. Since the real size of the detector is too small to obtain confident tally results in the TDMC simulations, the detector size is enlarged to cover the active core region at each intersection of air gaps. The tally region of the detector is shown in Figure 3. In the detector regions, the detector signals are tallied as a sum of $(n, \alpha)$ and $(n, p)$ reaction rates while the neutron simulation is conducted as if the detectors are filled with air to prevent them distorting the MC neutron tracking.

3.2. Searching the Optimum Detector Positions. To verify the feasibility of the devised method, $\overline{\alpha_{\text {est }}}\left(\mathbf{r} \mid t_{s}\right)$ is estimated at the two detector positions which are marked as optical fibers $\# 1$ and \#2 in Figure 1. $\overline{\alpha_{\text {est }}}\left(\mathbf{r} \mid t_{s}\right)$ at each detector position is compared with $\alpha_{\text {ref }}$ calculated by the $\alpha$-iteration method and $\alpha_{\exp }\left(\mathbf{r} \mid t_{s}\right)$ which is estimated by the exponential regression of experimental detector signals. Note that comparison results for optical fiber \#3 are omitted because its detector signals might be contaminated with gamma-ray induced by high energy neutron sources. $\overline{\alpha_{\text {est }}}\left(\mathbf{r} \mid t_{s}\right)$ is estimated with 100 replicas of TDMC simulation using 1,000,000 histories and $0.1 \mathrm{~ms}$ time bin up to $5 \mathrm{~ms}$. $\alpha_{\text {ref }}$ is calculated by the $\alpha$-iteration method with 100,000 histories and 100 active iterations. ENDF/B-VII.1 cross section libraries are used for both calculations.

Figure 4 shows comparison results for the two detector positions. The solid lines and the dashed lines are the TDMC and experimental results at detector positions. The value of $\alpha_{\text {ref }}$ is estimated to be 1950.0 with its standard deviation of 2.0. From the figure, one can see that the $\alpha$ estimates converge to the reference value with different convergence rates depending on their positions. Also one can see a discrepancy of the initial convergence trends of detector \#2 between the TDMC and experimental results, which can be attributed to a difference of detector signal yields sensitive to neutron energy range. The convergence times detected by (5) using the TDMC tally results are $0.8 \mathrm{~ms}$ for detector \#1 and $0.4 \mathrm{~ms}$ for detector \#2, whereas those from the experiments are $1.5 \mathrm{~ms}$ for detector \#1 and $1.1 \mathrm{~ms}$ for detector \#2. Although the convergence times estimated from the TDMC calculation differ from the experiments' by $0.7 \mathrm{~ms}$ for both detectors due to initial effects of the higher-mode components, it is noteworthy that their differences between the two detector positions are the same as $0.4 \mathrm{~ms}$. This implies that the proposed method based on the TDMC calculation can predict quite well the sensitivity of the $\alpha$ convergence time depending on detector positions.

The TDMC $\alpha$ estimations are conducted for all possible detector positions in the air gaps between assemblies to search the optimum detector positions. Figure 5 shows the convergence time and amplitude of neutron signal at each candidate position. The white colored positions in the convergence time map are where the $\alpha$ estimates do not converge until $4.0 \mathrm{~ms}$. Both the convergence time and the neutron signal map show that the polyethylene moderator regions adjacent to fuel region converge faster and give higher neutron signals than other regions. It is expected to obtain more reliable detector signals for the PNS $\alpha$ measurement at these optimum detector positions. 

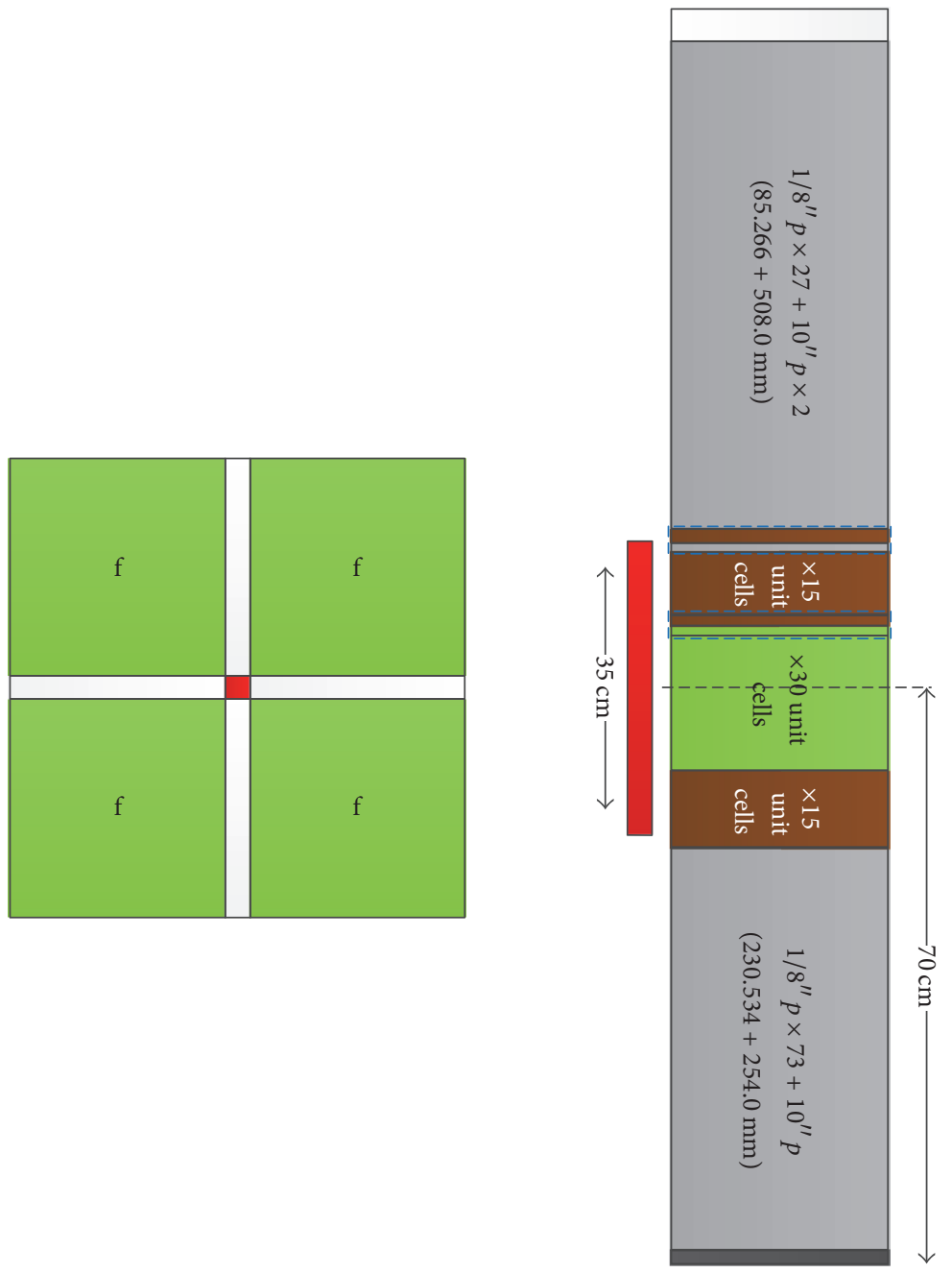

FIGURE 3: Tally region of the optical fiber detector (red region).

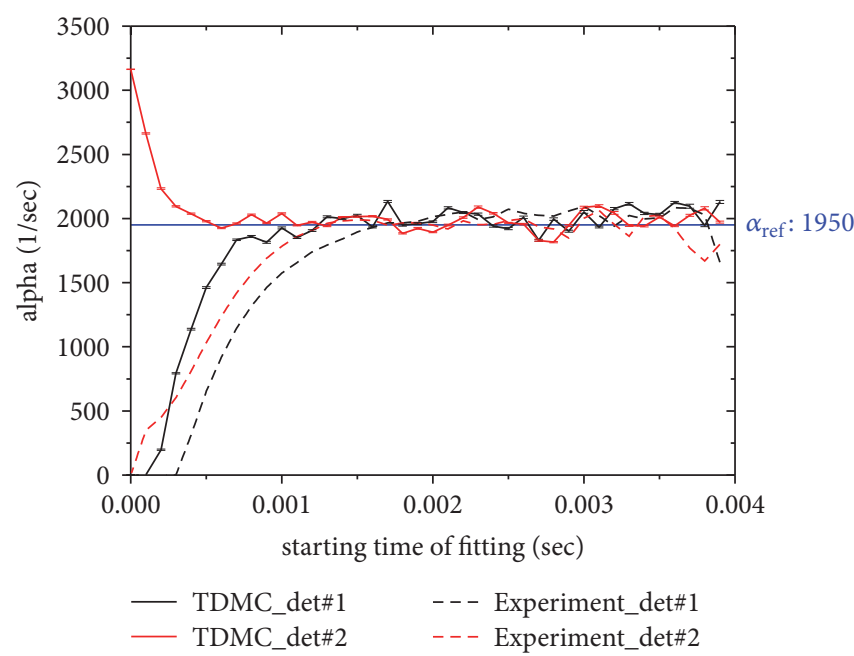

FIGURE 4: Comparisons of $\alpha$ estimates from TDMC and experimental data. 

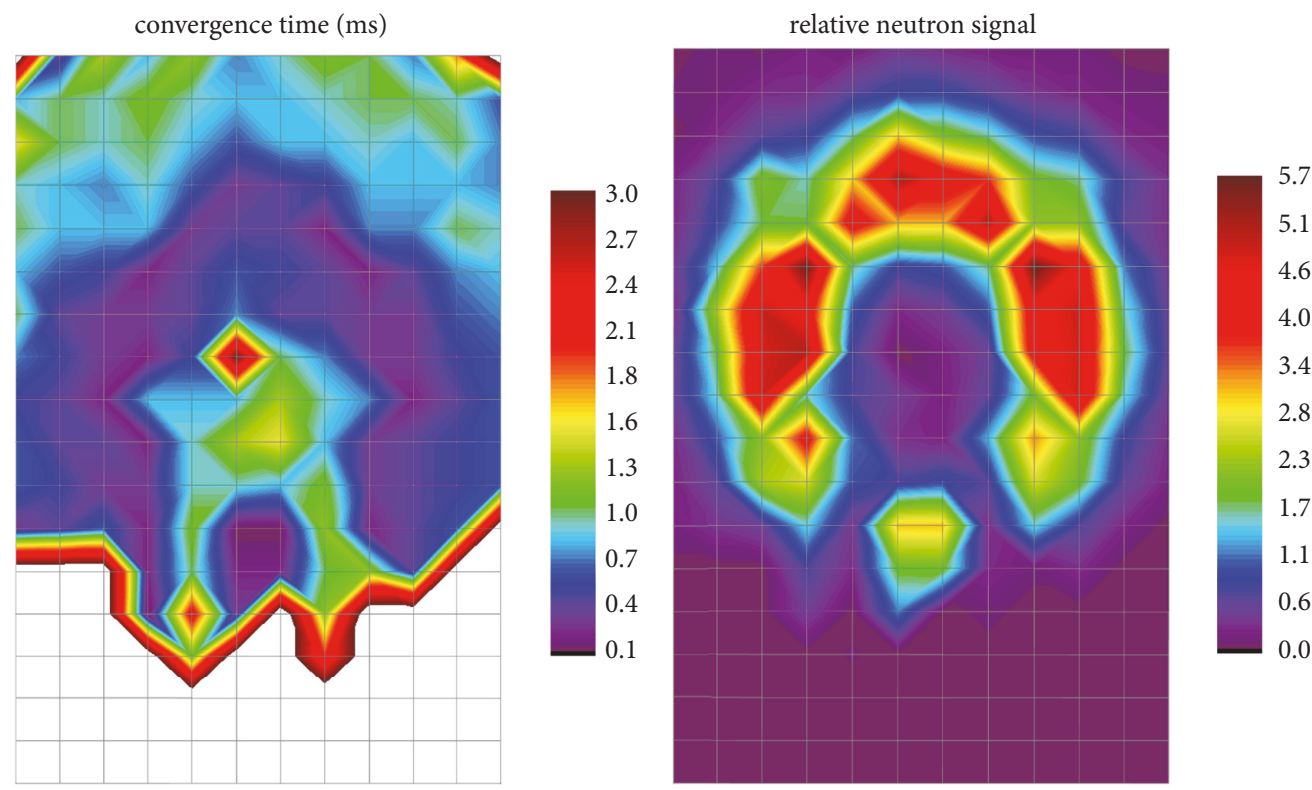

FIGURE 5: Convergence time and amplitude of neutron signal maps.

\section{Conclusions}

A simple method to determine an effective detector position for the PNS $\alpha$ measurement is proposed by comparing signal amplitudes at different detector positions estimated by the TDMC neutron transport calculations when their $\alpha$ estimates by the slope fitting are converged. The developed method is implemented in McCARD and applied to case 6 core in the KUCA Pb-Bi-zoned ADS experimental benchmarks. From the comparisons with experimental results, it is shown that the TDMC calculation predicts the $\alpha$ convergence time quite well. The proposed method provides the $\alpha$ convergence time map and the corresponding signal amplitude map for case 6 core, which can be used to determine effective detector positions and to validate experimental results in the PNS $\alpha$ measurement.

\section{Disclosure}

The authors have presented an earlier version of this research to RPHA17 (the Reactor Physics Asia 2017) conference, Chengdu, China, August 24-25, 2017.

\section{Conflicts of Interest}

The authors declare that they have no conflicts of interest.

\section{Acknowledgments}

This research is supported by the Brain Korea 21 Plus Project (no. 21A20130012821).

\section{References}

[1] H. A. Abderrahim, J. Galambos, Y. Gohar et al., "Accelerator and Target Technology for Accelerator Driven Transmutation and
Energy Production," DOE white paper on ADS, vol. 1, no. 1, pp. $1-23,2010$.

[2] D. De Bruyn et al., in Proceedings of the ICAPP 2016, San Francisco, CA, USA, 2016.

[3] X. Yan, L. Yang, X. Zhang, and W. Zhan, "Concept of an accelerator-driven advanced nuclear energy system," Energies, vol. 10, no. 7, article no. 944, 2017.

[4] A. Gandini and M. Salvatores, "The physics of subcritical multiplying systems," Journal of Nuclear Science and Technology, vol. 39, no. 6, pp. 673-686, 2002.

[5] K. Kobayashi and K. Nishihara, "Definition of subcriticality using the importance function for the production of fission neutrons," Nuclear Science and Engineering, vol. 136, no. 2, pp. 272-281, 2000

[6] R. Soule, W. Assal, P. Chaussonnet et al., "Neutronic Studies in Support of Accelerator-Driven Systems: the MUSE Experiments in the MASURCA Facility," Nuclear Science and Engineering, vol. 148, no. 1, pp. 124-152, 2004.

[7] C. H. Pyeon, M. Hervault, T. Misawa, H. Unesaki, T. Iwasaki, and S. Shiroya, "Static and kinetic experiments on acceleratordriven system with $14 \mathrm{MeV}$ neutrons in Kyoto university critical assembly," Journal of Nuclear Science and Technology, vol. 45, no. 11, pp. 1171-1182, 2008.

[8] C. H. Pyeon, T. Misawa, J.-Y. Lim et al., "First injection of spallation neutrons generated by high-energy protons into the kyoto university critical assembly," Journal of Nuclear Science and Technology, vol. 46, no. 12, pp. 1091-1093, 2009.

[9] B. E. Simmons and J. S. King, "A Pulsed Neutron Technique for Reactivity Determination," Nuclear Science and Engineering, vol. 3, no. 5, pp. 595-608, 1958.

[10] G. R. Keepin, Physics of nuclear kinetics, Addison-Wesley Pub. Co, MA, USA, 1965.

[11] F. R. N. McDonnell and M. J. Harris, "Pulsed-source experiments in a reflected coupled-core reactor-I. reactivity measurements," Journal of Nuclear Energy, vol. 26, no. 3, pp. 113-128, 1972. 
[12] É. A. Stumbur, A. G. Shokod’ko, V. I. Zhuravlev, I. P. Matveenko, and Z. N. Milyutina, "Combined pulsed method of measuring high reactivities for reactors with reflectors," Soviet Atomic Energy, vol. 36, no. 3, pp. 224-228, 1974.

[13] T. Suzaki, "Subcriticality Determination of Low-Enriched UO2 Lattices in Water by Exponential Experiment," Journal of Nuclear Science and Technology, vol. 28, no. 12, pp. 1067-1077, 1991.

[14] C.-M. Persson, P. Seltborg, A. Åhlander et al., "Analysis of reactivity determination methods in the subcritical experiment Yalina," Nuclear Instruments and Methods in Physics Research Section A: Accelerators, Spectrometers, Detectors and Associated Equipment, vol. 554, no. 1-3, pp. 374-383, 2005.

[15] C. H. Pyeon, Experimental Benchmarks on Thorium-Loaded Accelerator-Driven System at Kyoto University Critical Assembly, KURR-TR(CD)-48, Research Reactor Institute, Kyoto University, 2015.

[16] C. H. Pyeon, M. Yamanaka, T. Endo, W. F. G. van Rooijen, and G. Chiba, "Experimental benchmarks on kinetic parameters in accelerator-driven system with $100 \mathrm{MeV}$ protons at Kyoto University Critical Assembly," Annals of Nuclear Energy, vol. 105, pp. 346-354, 2017.

[17] D. E. Cullen, C. J. Clouse, R. Procassini, and R. C. Little, "Static and Dynamic Criticality: Are They Different?" Tech. Rep. UCRL-TR-201506, 2003.

[18] B. L. Sjenitzer and J. Eduard Hoogenboom, "Dynamic monte carlo method for nuclear reactor kinetics calculations," Nuclear Science and Engineering, vol. 175, no. 1, pp. 94-107, 2013.

[19] N. Shaukat, M. Ryu, and H. J. Shim, "Dynamic Monte Carlo transient analysis for the Organization for Economic Co-operation and Development Nuclear Energy Agency (OECD/NEA) C5G7-TD benchmark," Nuclear Engineering and Technology, vol. 49, no. 5, pp. 920-927, 2017.

[20] T. E. Booth, "A Weight (Charge) Conserving ImportanceWeighted Comb for Monte Carlo," LA-UR-96-0051, Los Alamos National Laboratory, NM, USA, 1996.

[21] H. J. Shim, B. S. Han, J. S. Jung, H. J. Park, and C. H. Kim, "McCARD: Monte Carlo code for advanced reactor design and analysis," Nuclear Engineering and Technology, vol. 44, no. 2, pp. 161-176, 2012.

[22] C. H. Pyeon, Experimental Benchmarks of Neutronics on Solid $\mathrm{Pb}-\mathrm{Bi}$ in Accelerator-Driven System with $100 \mathrm{MeV}$ Protons at Kyoto University Critical Assembly, KURRI-TR-447, Research Reactor Institute, Kyoto University, 2017.

[23] H. J. Shim, S. H. Jang, and S. M. Kang, "Monte Carlo Alpha Iteration Algorithm for a Subcritical System Analysis," Science and Technology of Nuclear Installations, vol. 2015, pp. 1-7, 2015.

[24] S. Nakamura, Computational methods in engineering and science, Wiley-Interscience [John Wiley \& Sons], New YorkLondon-Sydney, 1977.

[25] J. S. Hendricks, Tech. Rep., LA-UR-08-2216, Los Alamos National Laboratory, NM, 2008.

[26] T. Yagi, H. Unesaki, T. Misawa et al., "Development of a small scintillation detector with an optical fiber for fast neutrons," Applied Radiation and Isotopes, vol. 69, no. 2, pp. 539-544, 2011. 

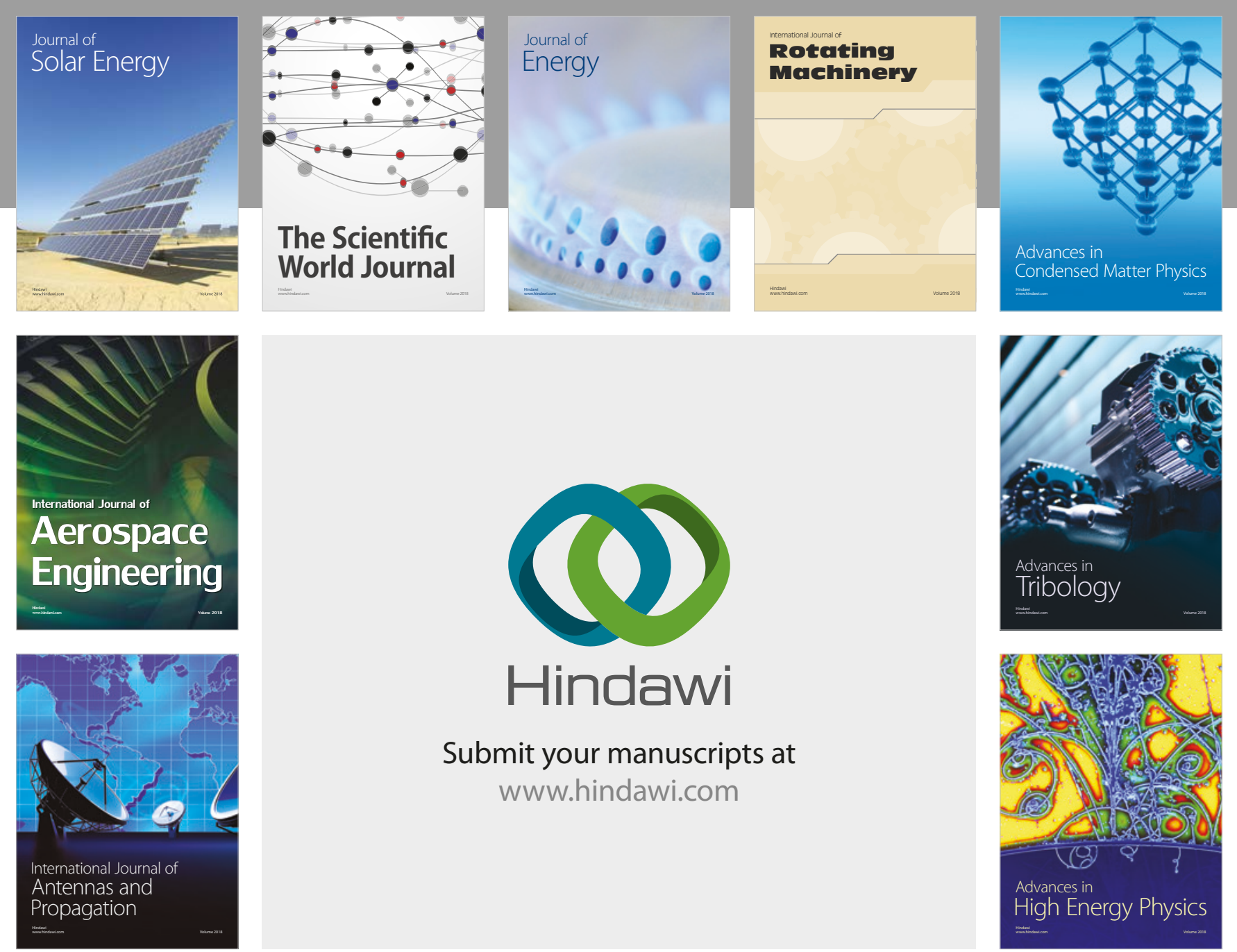

Submit your manuscripts at

www.hindawi.com
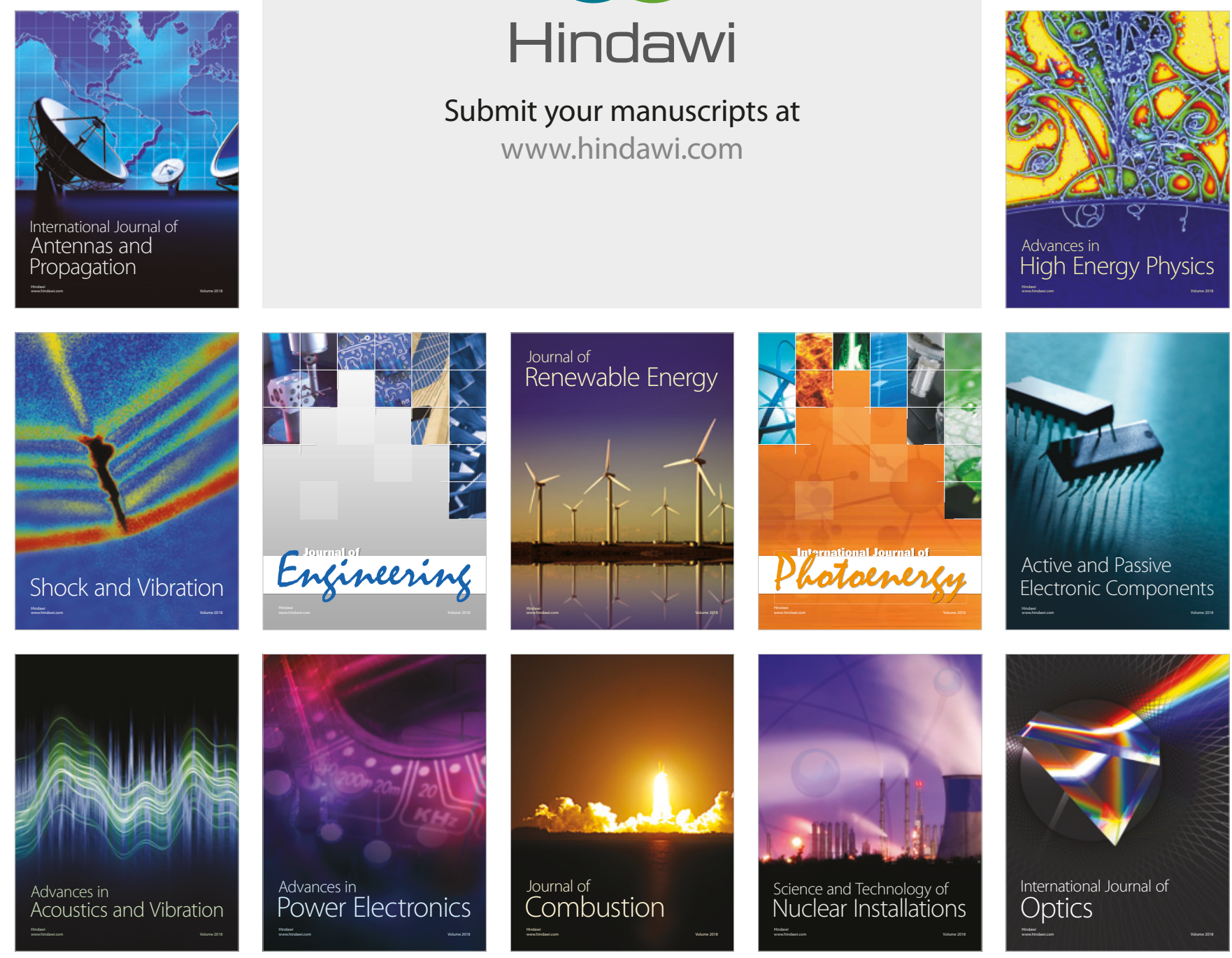